\title{
Hepatocellular Carcinoma with Direct Invasion to the Stomach
}

\author{
Hiromu Inoue, Yukihisa Sawada, Kaori Ochiai, Hideaki Honda, Junko Murayama, \\ Yasutaka Kudo, Yoshiyuki Nakashima, Noriyoshi Sagihara, Hiroyuki Miyatani, \\ Ikuo Nakamura and Yukio Yoshida
}

\begin{abstract}
A 71-year-old man was admitted to our hospital with abdominal pain. Hepatocellular carcinoma (HCC) had been diagnosed 2 years earlier and he had undergone 7 courses of intra-hepato-arterial chemotherapy (IHAC). We performed gastrointestinal fiberscopy and identified a massive protrusion on the lesser curvature. Abdominal contrast-enhanced computed tomography revealed multiple hepatic masses and an extrahepatic enlarged mass with invasion to the pancreas and stomach. A specimen for endoscopic biopsy revealed adenocarcinoma that stained positive for $\alpha$-fetoprotein. Gastrointestinal bleeding resulting from direct invasion of HCC is unusual.
\end{abstract}

Key words: hepatocellular carcinoma, gastrointestinal hemorrhage, direct invasion

(DOI: 10.2169/internalmedicine.46.6048)

\section{Introduction}

Intra-hepato-arterial chemotherapy (IHAC) is useful treatment for hepatocellular carcinoma (HCC), but advanced $\mathrm{HCC}$, in particular an extrahepatic lesion including a lymph node is uncontrollable. Here, we describe a case of hepatocellular carcinoma with the direct invasion to the stomach. It was suggested that the extrahepatic lymph node was enlarged and had invaded into the gastrointestinal (GI) tract.

\section{Case Report}

A 71-year-old man was admitted to our hospital with abdominal pain. Liver cirrhosis associated with hepatitis $\mathrm{C}$ virus infection had been diagnosed about 5 years earlier at another hospital. Abdominal computed tomography (CT) after 3 years revealed a $5-\mathrm{cm}$ mass in segment IV that had invaded the adjacent portal vein. Unresectable hepatocellular carcinoma (HCC) was diagnosed. Intra-hepato-arterial chemotherapy (IHAC) comprising 5-fluorouracil and cisplatin every 3 months was initiated. The patient had just undergone the seventh course of IHAC 2 months before admis- sion, and chemotherapy was well tolerated. However, he noticed abdominal pain in the left upper quadrant after discharge, 1 month before admission. The symptoms were gradually exacerbated and oral intake became impossible. He had lost 5-6 kg body weight in the preceding months. The patient was therefore readmitted for detailed reevaluation. He had undergone coronary artery bypass grafting at the age of 52 years old, and medication included aspirin. He had a history of smoking 10 cigarettes/day for 45 years, and infrequent alcohol intake. There was history of intravenous drug use. On physical examination, the patient was $155 \mathrm{~cm}$ tall and weighed $50 \mathrm{~kg}$. Blood pressure was $156 / 93 \mathrm{mmHg}$ and heart rate was 90 beats/minute. Examination of the lungs and heart revealed no abnormalities. The abdomen was distended with evidence of ascites and tenderness. A poorly movable mass was palpable extending $5 \mathrm{~cm}$ below the xyphoid process. All distal pulses were palpable and no peripheral edema was present. Laboratory studies showed severe normocytic anemia (erythrocyte count, $256 \times 10^{4} / \mu \mathrm{l}$; hemoglobin, $7.9 \mathrm{~g} / \mathrm{dl}$; hematocrit, 18.5\%). The level of $\alpha$ fetoprotein was markedly elevated at $45,630 \mathrm{ng} / \mathrm{ml}$. The laboratory data on admission is shown in Table 1. Upper gastrointestinal fiberscopy revealed a massive protrusion 
Table 1. Laboratory Finding on Admission

\begin{tabular}{ll}
\hline \multicolumn{1}{c}{ peripheral blood } \\
red blood cell & $256 \times 10 / \mu \mathrm{l}$ \\
hemoglobin & $7.9 \mathrm{~g} / \mathrm{dl}$ \\
platelet & $29.4 \times 10 / \mu \mathrm{l}$ \\
white blood cell & $7660 / \mu \mathrm{l}$ \\
\multicolumn{1}{c}{ blood chemistry } & \\
total protein & \\
albumin & $6.6 \mathrm{~g} / \mathrm{dl}$ \\
T-bilirubin & $2.7 \mathrm{~g} / \mathrm{dl}$ \\
AST & $0.5 \mathrm{mg} / \mathrm{dl}$ \\
ALT & $66 \mathrm{U} / \mathrm{ml}$ \\
LDH & $16 \mathrm{U} / \mathrm{ml}$ \\
ALP & $247 \mathrm{mU} / \mathrm{ml}$ \\
$\gamma \mathrm{GTP}$ & $242 \mathrm{mU} / \mathrm{ml}$ \\
Serum urea nitrogen & $157 \mathrm{mU} / \mathrm{ml}$ \\
Creatinine & $32 \mathrm{mg} / \mathrm{dl}$ \\
alphafetoprotein & $2.1 \mathrm{mg} / \mathrm{dl}$ \\
$\quad$ coagulation system & $45630 \mathrm{ng} / \mathrm{ml}$ \\
prothrombine time & \\
&
\end{tabular}

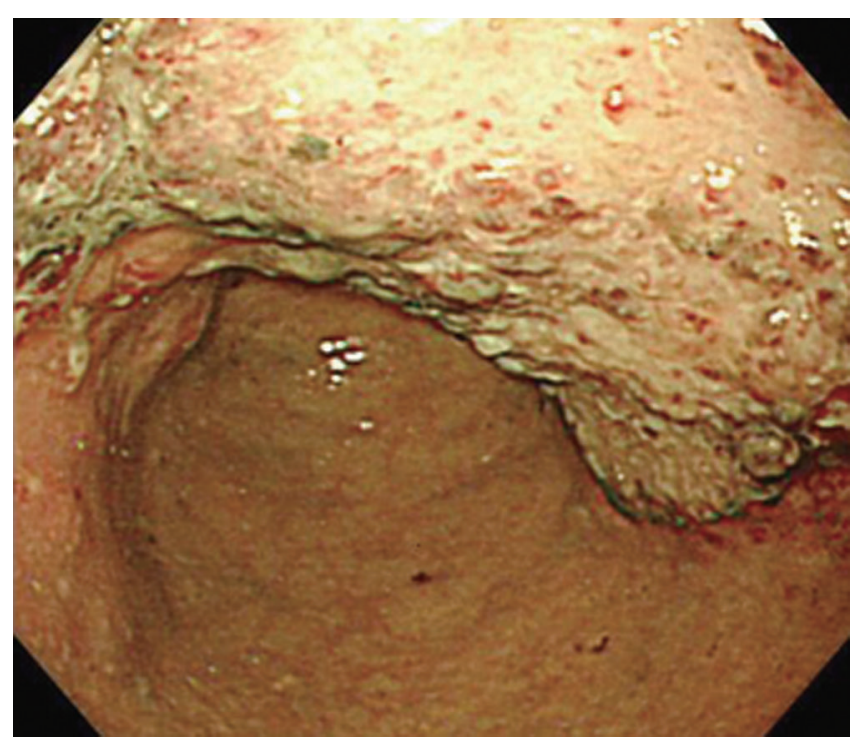

Figure 1. Upper gastric endoscopy showing a massive lesion on the lesser curvature from the upper body to the antrum of the stomach.

containing necrotic tissue on the surface, from the lesser curvature to the posterior wall of the body of the stomach (Fig. 1). No esophageal varices were present. Contrastenhanced CT revealed an enlarged lymph node that had invaded directly into the pancreas and posterior wall of the stomach (Fig. 2). Omeprazole was administered intravenously at $40 \mathrm{mg} / \mathrm{day}$, and red blood cells (4 units) was transfused. Abdominal pain was relieved using pentamidine.

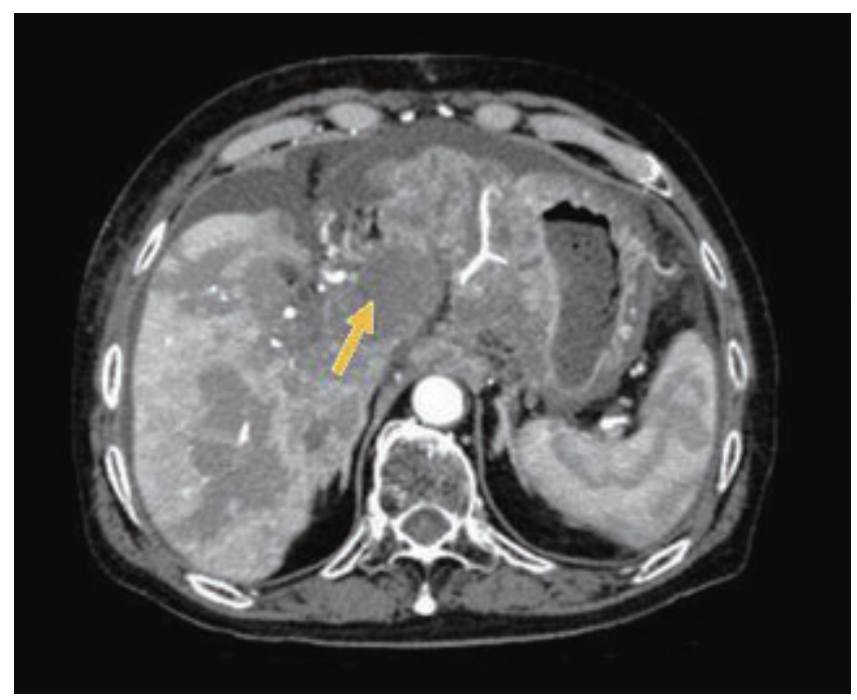

Figure 2. Contrast-enhanced computed tomography on admission shows direct invasion to the stomach. Extrahepatic lymph nodes (arrow) have grown rapidly and invaded the stomach and pancreas.

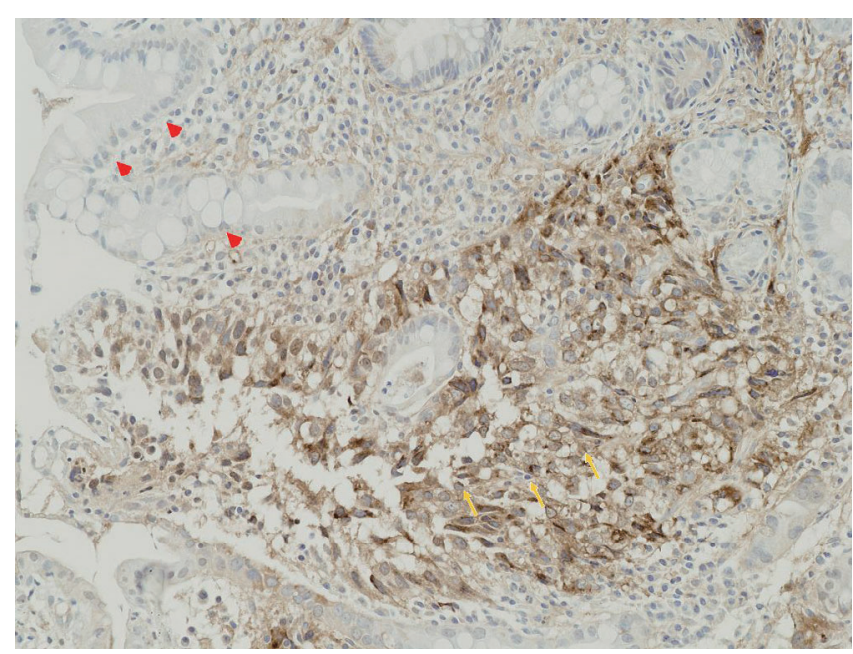

Figure 3. Pathologic examination revealed moderately differentiated HCC invading the gastric mucosa, and showed positivity for $\alpha$-fetoprotein on immunological staining (arrows). But the original gastric mucosa (arrowheads) was negative.

After oral intake was restarted, hemoglobin levels were stable and abdominal pain remained under control. Pathological examination revealed moderately differentiated adenocarcinoma with positive immunological staining for $\alpha$-fetoprotein (Fig. 3). The patient was transferred to another hospital for palliative care on hospital day 7 .

\section{Discussion}

Gastrointestinal bleeding is a common complication in patients with advanced HCC, and is mostly due to variceal rupture. Gastrointestinal bleeding in hepatocellular carci- 


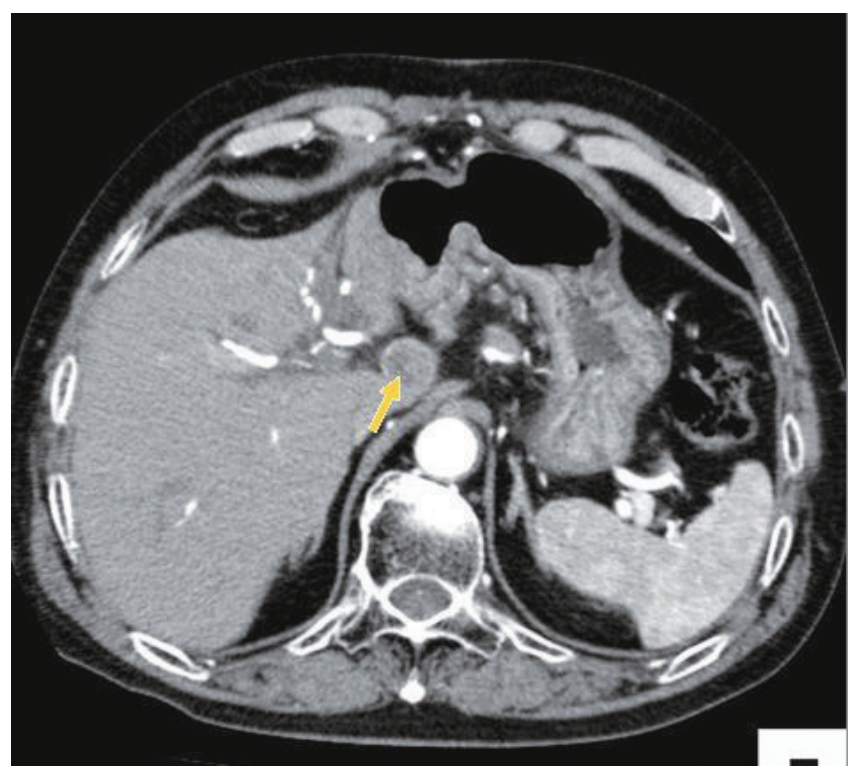

Figure 4. Computed tomography 6 months before admission, showing that the intrahepatic lesion is under control, but the lymph node (arrow) is enlarged.

noma is uncommon, and most cases are found on postmortem examination. Chen et al reported that $2 \%$ of HCCs display GI tract involvement (1). Among the 396 patients with hepatocellular carcinoma who presented to their hospital in Taiwan between 1982 and 1988, 8 cases with HCC had spread to the GI tract; they described the clinical presentation and the course of the disease. The median time between the diagnosis of primary tumor and gastrointestinal tract involvement was 4.5 months. In addition, almost all cases were advanced stage of HCC at diagnosis. It was suggested that the cases of GI tract involvement were due to delayed diagnosis. Compared to their report, the present case was diagnosed as liver cirrhosis with hepatitis $\mathrm{C}$ virus infection 5 years and HCC was detected 3 years before GI tract involvement. In Japan, to date, untreatable or an advanced state of $\mathrm{HCC}$ at the initial diagnosis is rare because the screening system for chronic hepatitis with hepatitis C or B virus infection is developed. Thus, we think that the rate of GI involvement must be less than that of the report of Chen et al. IHAC is performed in most cases of direct invasion to the stomach (3-5), and may induce tumor necrosis and subcapsular tumor adhesion to the GI serosa (2). In the present case, swelling of an extrahepatic lymph node rather than hepatoma had invaded directly to the stomach. Some authors have reported hematogeneous HCC spread to the stomach (6-8), suggesting that metastasis is due to portal vein thrombosis and hepatofugal blood flow to the GI tract. However, direct invasion to the gastrointestinal tract via lymph nodes is rare. In this patient, HCC that had invaded the adjacent portal vein was evaluated as unresectable 2 years earlier. IHAC was initiated and proved effective for the primary tumor, but lymph nodes on the porta hepatica were uncontrollable. The previous CT, performed 6 months (Fig. 4) before admission, revealed lymph nodes $2 \mathrm{~cm}$ in diameter on the porta hepatica. In addition, several lymph nodes were noted along the lesser curvature of the stomach. Anti-tumor drugs were delivered to the hepatic tissue effectively, but not to the extrahepatic lymph nodes. These lymph nodes appear to have rapidly become enlarged in size, and invaded not only the pancreas and stomach, but also the left lobe of the liver. In general, the prognosis for patients with gastrointestinal tract involvement of HCC is very poor. Natsuizaka et al analyzed 65 patients with extrahepatic metastasis of HCC (9). In their report, 22 patients $(33.8 \%)$ displayed lymph node metastases, with a median survival of 7 months and a 1-year survival rate of $24.9 \%$. To date, no effective treatment is available for HCC with extrahepatic metastases, but some advanced cases have been successfully treated using oral administration of enteric-coated tegafur/uracil $(11,12)$. If hepatic reserve and performance status are good, systemic chemotherapy using oral administration could be considered. Lin et al described the prognoses for 11 cases with gastrointestinal tract metastasis in HCC, and almost all patients died within 5 months (10). They also reported endoscopic features and clinical courses of those patients. The endoscopic features included ulcerative tumors mimicking advanced cancer $(43 \%)$ and submucosal tumors (29\%). In conclusion, gastrointestinal involvement in HCC is uncommon. In the present case, it was suggested that extrahepatic lymph nodes enlarged rapidly and invaded the gastric wall. Considering systemic chemotherapy for extrahepatic metastasis is important, in addition to IHAC.

\section{References}

1. Chen LT, Chen CY, Jan CM, et al. Gastrointestinal tract involvement in hepatocellular carcinoma: Clinical, radiological and endoscopic studies. Endoscopy 22: 118-123, 1990.

2. Maruyama A, Murabayashi K, Hayashi M, et al. Hepatocellular carcinoma complicated by gastrointestinal hemorrhage caused by direct tumor invasion of stomach. J Hepatobiliary Pancreat Surg 6: 90-93, 1999.

3. Kumai T, Suzuki T, Kobayashi E, et al. A case of a metastatic hepatocellular carcinoma to the stomach. Review of Japanese literature. Jpn J Gastroenterol 88: 749-753, 1991.

4. Nicoll AJ, Ireton HJC, Crotty B. Gastrointestinal bleeding from hepatocellular carcinoma invading the stomach. J Gastroenterol Hepatol 9: 533-535, 1994.
5. Lynch P, Green L, Jordan PH, Graham DY. Gastrointestinal bleeding from hepatocellular carcinoma metastatic to the stomach presenting as bleeding multiple crateogenic ulcers. Am J Gastroenterol 84: 653-655, 1989.

6. Hashimoto M, Watanabe G, Matsuda M, Yamamoto T, Tsutsumi K, Tsurumaru M. Tumors of liver and pancreas. Case report: Gastrointestinal bleeding from a hepatocellular carcinoma invading the transverse colon. J Gastroenterol Hepatol 11: 765-767, 1996.

7. Chen CY, Lu CL, Pan CC, Chiang JH, Chang FY, Lee SD. Lower gastrointestinal bleeding from a hepatocellular carcinoma invading colon. J Clin Gastroenterol 25: 373-375, 1997.

8. Kalra TMS, Mangla JC, Schwartz S, Lee JCK. Hepatoma presenting as lower gastrointestinal bleeding. Am J Gastroenterol 67: 
485-488, 1997.

9. Natsuizaka M, Omura T, Akaike T, et al. Clinical features of hepatocellular carcinoma with extrahepatic metastases. J Gastroenterol Hepatol 20: 1781-1787, 2005.

10. Lin CP, Cheng JS, Lai KH, et al. Gastrointestinal metastasis in hepatocellular carcinoma: radiological and endoscopic studies of 11 cases. J Gastroenterol Hepatol 15: 536-541, 2000.

11. Ishikawa $T$, Ichida $T$, Ishimoto $Y$, et al. Complete remission of multiple hepatocellular carcinomas associated with hepatitis C virus-related decompensated liver cirrhosis by oral administration of enteric-coated tegafur/uracil. Am J Gastroenterol 94: 1682$1685,1999$.

12. Ishikawa $T$, Ichida $T$, Sugitani $S$, et al. Improved survival with oral administration of enteric-coated tegafur/uracil for advanced stage IV-A hepatocellular carcinoma. J Gastroenterol Hepatolol 16: 452-459, 2001.

(C) 2007 The Japanese Society of Internal Medicine http://www.naika.or.jp/imindex.html 